\title{
Prioritized Rail Corridor Asset Management
}

\author{
T. Selig ${ }^{1} \&$ J. Kasper ${ }^{2}$ \\ ${ }^{1}$ Optram Inc., Maynard, Masschussets, USA \\ ${ }^{2}$ MRO Software, Inc., Bedford, Masschussets, USA
}

\begin{abstract}
Seldom do railroads have the resources to maintain their infrastructure at such a level to ensure steady-state performance. More often they are faced with the decision of which maintenance actions should take priority, so that optimal safety and reliability are maintained under the burden of constrained resources. The option of additional resources is rarely feasible. Given these current operating realities, more and more railroads are finding that the solution lies in utilizing information technology to work more efficiently. By harnessing the vast amount of existing rail corridor data into a prioritized plan, and then assigning the work and monitoring the execution and results with software tools, many railroads are attempting to do more with less. The strategy, we define as Prioritized Rail Corridor Asset Management, not only results in steady-state asset performance under constrained resources but can even improve the asset condition and provide a positive return on investment.

This paper describes an information system developed to facilitate a Prioritized Rail Corridor Asset Management strategy. The enterprise-scale solution requires combining Optram's Railway Infrastructure Management $\left(\mathrm{ORIM}^{\mathrm{TM}}\right)$ information system with MRO Software, Inc. (MROI) strategic asset management information system $\left(\right.$ MAXIMO $\left.^{\circledR}\right)$. ORIM integrates existing rail corridor data sources with a linear model of the track network to continuously generate a prioritized work plan. The prioritized work plan is linked to MAXIMO where staff, materials, and equipment are managed. The results combined with an effective execution facilitate a Prioritized Rail Corridor Asset Management strategy.

Keywords: Railway Infrastructure Asset Management, Enterprise Asset Management, Linear Asset Management, Track Maintenance Management, Rail Management, Enterprise Information Systems.
\end{abstract}




\section{The Challenge}

Managing a reliable and safe rail corridor is typically performed with insufficient information and limited resources. Having simple to use and readily available asset location and condition information to prioritize the use of limited resources (people, material, equipment, and work windows) can dramatically affect the rail corridor reliability, safety and profitability. Compounding the problem is a continuous flood of data collected from activities such as track inspections, geometry car surveys, rail defect detectors, ground penetrating radar, rail profile measurements, video surveys, infrared tests, asset surveys, work records, train movement, wayside detectors and more. Converting this vast, continuous and varied stream of data into management intelligence to develop a prioritized plan to optimize maintenance-of-way resources is a significant challenge.

Successful applications of prioritized rail corridor asset management can have significant and lasting impact. It has been shown at Amtrak and Conrail [1] and at Network Rail and First Engineering [2] that without additional capital investment a $10 \%$ annual savings in maintenance expense could be saved by targeting timing and location of capital and maintenance operations and resources [1]. Higher returns can be realized when capital investments are also made in the infrastructure.

\section{Rail Corridor characteristics}

\subsection{Rail Corridors as a linear asset}

Rail corridors can best be characterized as comprised of a network of interrelated linear or "continuous" assets. The linear asset is similar to other capital-intensive assets - such as facilities and fleets of vehicles - in that they are expensive to build and maintain, and have a direct impact on rail service reliability, safety and profitability. On the other hand, there are important differentiations for linear assets:

- They are geographically dispersed, with few or no obvious physical characteristics that distinguish one asset from another, other than their relative location

- Maintenance work and component wear is distributed over varying portions of the asset in disperse and non-discrete locations along the asset;

- Linear assets are comprised of an interrelated series of components (rail, sleepers/ties, fasteners, ballast, subballast) that vary along the length of the asset

- The performance of a part of one asset may affect part of another (e.g., a wet spot could increase rail defect rate, bridges transitions, switch and crossings, curves)

- Work is performed on a portion of an asset on various components

- Use characteristics (speed, tonnage, etc.) vary by asset location and component 
In short, the unique continuous and interrelated nature of linear assets requires a specialized approach to develop and manage a safe, cost-effective and reliable maintenance-of-way plan.

\subsection{Impact of Rail Corridor Asset Performance}

A railway system is made up of vehicles, facilities, and rail corridor assets. A failure in a vehicle asset can disrupt a single carload and possibly a comprising train making a minor impact on a corridor's throughput. Similarly a failure in facility typically has a minor impact on the systems overall performance. On the contrary, the rail corridor, as a linear asset, acts like a pipeline. A disruption of just a small portion of the rail corridor creates a dramatic impact throughout the asset [3]. In areas of moderate traffic, a single problem in a 2-track configuration can cut throughput by $50 \%$ or more. In addition, when the track is operating at capacity, disruptions typically result in lost and unrecoverable service revenue.

\section{System for Prioritized Rail Corridor Asset Management}

A system for Prioritized Rail Corridor Asset Management is comprised of two integrated information management systems (Figure 1). Optram's ORIM Railway Infrastructure Management (ORIM) system uses condition, traffic, and work data to develop a prioritized work plan. The prioritized plan is sent to MROI's MAXIMO enterprise asset management system to manage and monitor the maintenance-of-way work.

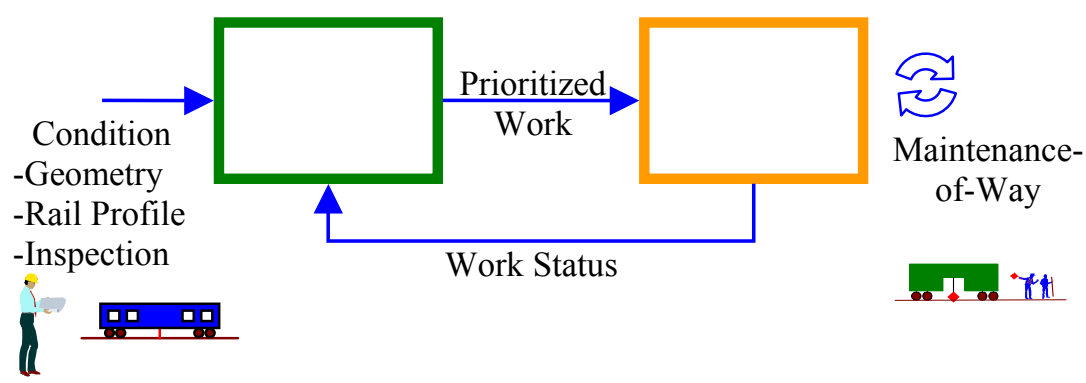

Figure 1: $\quad$ Prioritized Rail Corridor Asset Management.

\subsection{Optram's Railway Infrastructure Management (ORIM) System for Prioritization}

Optram's Railway Infrastructure Management (ORIM) System is designed to analyze and correlate multiple data-sets about the rail corridor to produce a 
prioritized plan of maintenance and capital corridor work. Optram, in collaboration with Amtrak, has been continuously developing this prioritized approach since 1997. Over that time Amtrak has continuously lead in the development and adoption of technology and solutions that incorporate prioritized rail asset management in Amtrak's system called Infrastructure Information Management System [4].

ORIM uses existing data about rail corridor configuration, condition, usage and maintenance to develop a prioritized plan. The rail corridor configuration includes the position and relation of assets along the right-of-way. Examples of condition data include track geometry, rail profile, rail defects, track exceptions, and visual inspections. Usage data examples include dynamic and static loads, wheel condition, and train movements. Work includes type of labor, materials and equipment use to maintain the right-of-way.

The Track Maintenance Management White Paper [3] paper describes ORIM as a Track Maintenance Management (TMM) system. It describes TMM systems as assisting rail organizations developing answers to the fundamental question of "What should I work on and why?" TMM systems collect and analyze condition, usage, work and performance data and relate the results to the assets located in the right-of-way. Performance data is derived from the degradation of condition over time and the effectiveness of repair work.

Optram's ORIM integrates existing rail corridor data sources and aligns it with a linear model of the track network. This integrated model is then analyzed to develop a prioritized work plan, to include near term and longer term maintenance work, including capital planning. As the work flow progresses, the status and performance of the work is fed back to ORIM to again refine the prioritized plan.

When freight and passenger rail organizations use Optram's Railway Infrastructure Management (ORIM) system they are able to shift their day-to-day work concentration:

- From generalized, reactive repairs to proactive focused repairs

- From annual production cycles to TMM-enabled targeted programs

Both approaches address root-causes, significantly reducing the expense of repeated repairs.

Heide et al. [1] showed in a substructure application of TMM that without additional capital investment, a 10\% annual savings in maintenance expense was possible by better targeting maintenance operations, and deploying resources to go only where and when they are needed [1]. Higher returns were realized when capital investments were made in the infrastructure.

\subsubsection{ORIM's return-on-investment, Scotland case study}

During the summer of 2002 a 3-month project was undertaken of the Optram Right-of-way Infrastructure Management (ORIM) product with an aim of demonstrating the potential for $10 \%$ or more improvement of maintenance efficiencies. In 2004 the pilot project covered 47 route miles of a double-track 
commuter passenger rail line. In 2003 the project was extended to include data for a 58-mile section of an intercity main line track.

The areas of track studied under the project were high priority lines that struggle with expensive track related train delays. The delay penalties mount up quickly considering that a 15-minute delay of a single train results in a penalty of $£ 2670$. With the possible advent of an additional 30 trains per day on the line, similar delays could amount to daily delay penalties in excess of $£ 80,000$.

Working with the customer, the project team identified an initial list of 10 areas where ORIM can be used to reduce maintenance costs and time delays. From this list the team selected three areas to show reduced maintenance costs and time delays:

1. Areas of rough track geometry,

2. Areas of repeat rail defects, and

3. Information-sharing and communication.

These three areas were selected because they represent: a range of savings-types, data was available within the project time and budget constraints and they provided edifying examples of the potential savings obtainable through ORIM use.

The project results concluded by using 3 of the 10 savings areas over the $1000 \mathrm{~km}$ of track the system would provide:

1. a strategic advantage for the maintenance contractor through delivery of improved reliability,

2. saving at least $£ 10 \mathrm{M}$ per year through improved understanding and control of track infrastructure, more effective maintenance, reduced delay penalties and improved work window productivity [2].

\subsection{MROI's Enterprise Asset Management System}

MROI's MAXIMO system provides a unified approach to managing all classes of assets, across the enterprise. MAXIMO provides real-time life cycle asset management, from sourcing through final disposal of assets. In addition, MAXIMO includes management of the work flow to maintain the assets.

Once the ORIM "engineering assessment" of asset condition and historical performance is complete, MAXIMO manages the logistics of assigning the steps to complete the work.

\section{Modelling the Rail Corridor}

ORIM develops its prioritized plan by relating multiple data sources about the rail corridor. The linear characteristic of the corridor requires a unique model to manage and interpret the continuous nature of related information. ORIM correlates data by the relative position within the corridor but without regard to discreet features. This type of data relation called "continuous linear relation" and is in contrast to the more typical relation of data by discrete "segmenting".

Segmenting of the track network breaks the rail network into discrete segments where data is grouped into buckets with areas defined by corridor 
features (e.g., tangent and curve track, bridge transitions, areas of common track components) or buckets defined by even sizes (1/8 mile segments, $1-\mathrm{km}$ segments). Segmenting often groups data that should not be correlated and thus blurs the interpretation of that data.

Figure 2 is an example ORIM track layout and work diagram. The figure shows work performed on the "South" track, which is represented by horizontal lines on the bottom half of the figure. The horizontal axis indicates where the work starts and ends (marked by the $\mathrm{km} /$ Mile Post markers on the top of the figure). The vertical axis represents when the work is preformed (marked by left hand date scale showing month and year).

It is apparent that work is performed regardless of the placement of such features as switches and bridges.

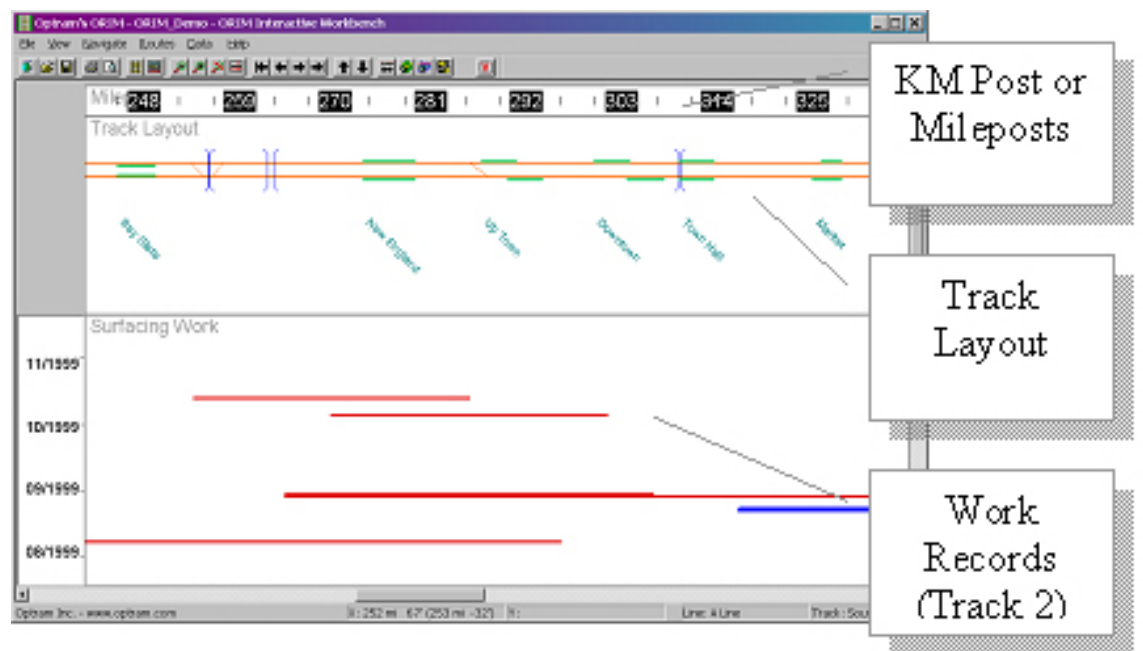

ORIM is protected under US patent 5,978,717

Figure 2: $\quad$ Rail Corridor Assets Model.

ORIM's use of “continuous linear relation” provides the ability to:

- Accurately pinpoint problem locations

- Avoid blurring of unrelated data

- Interpret multiple issues from a common data set

- Add new linear data sets without re-segmenting

\section{Technology to prioritize}

To create a prioritized plan the following elements are needed:

- A system that captures and integrates the flood of data collected on a rail corridor.

- A system that schedules, assigns, combines workflow and monitors the people, equipment, and material to complete the asset maintenance and 
renewal work. This includes where on the linear asset the work is performed.

- A simple and efficient presentation of portraying the large data set as information.

To provide this solution Optram and MROI combine Optram's linear asset management information system with MROI's strategic asset management information system. These elements combined with effective execution can turn all the data into a prioritized scheme for optimizing resources to maintain the highest throughput, reliability, and safety.

\section{ORIM - MAXIMO Integration}

Together, ORIM and MAXIMO systems provide:

- Targeted, proactive maintenance

- Accurate long-term maintenance planning based upon actual asset condition, work history and performance

- Automatic generation of pre-planned work orders and associated logistic support elements

Close integration of ORIM and MAXIMO systems also streamlines the asset analysis/work-order maintenance process, improves user productivity, reduces potential errors between these two critical functions, and lowers railroad operators' overall IT support costs.

Asset, condition indicators and work-order data is exchanged continuously between the ORIM and MAXIMO to facilitate a focused and streamlined Track Maintenance Management process. Further discussion of the ORIM and MAXIMO relation is described in [3].

The ORIM / MAXIMO integration streamlines the asset analysis and workorder maintenance lifecycle process, improving user productivity, reducing potential errors between these two critical functions, and lowering railroad operators' overall IT support costs.

\section{Conclusion}

Managing a reliable and safe rail corridor is challenge. Having simple and readily available information to prioritize the use of limited resources (people, material, equipment, and work windows) can dramatically affect the rail corridor reliability, safety and profitability. Using a Prioritized Rail Corridor Management system can convert the vast, continuous and varied stream of rail corridor data into management intelligence required to continuously manage the safest, least expensive and most reliable rail corridor under constrained resources. 


\section{References}

[1] Walter Heide, Conrad Ruppert, Ernest T. Selig and Willem Ebersöhn, Engineering Approach to Track Substructure Management on Conrail and Amtrak, AREA Annual Meeting presentation, March26, 1996.

[2] "Scotland Alliance ORIM Pilot Project Report, A Summary Report for the Railtrack and First Engineering Scotland Alliance", May 6, 2003

[3] "Track Maintenance Management Executive White Paper", Optram Inc., May 2003, www.optram.com

[4] Dr. Willem Ebersöhn, Conrad J. Ruppert, Jr., "Railway Infrastructure Maintenance Management at Amtrak", UK Institute of Mechanical Engineers, September 2002.

[5] ORIM is protected under patent 5,978,717 